\title{
Using a textual representational level component in the context of discourse or dialogue generation
}

\author{
Franck Panaget* \\ Centre National d'Etudes des Télécommunications (CNET) \\ $\mathrm{LAA} / \mathrm{TSS} / \mathrm{RCP}$ \\ Route de Trégastel - 22300 Lannion - France \\ email: panaget@lannion.cnet.fr / tel: +33 96.05.28.52 / fax: +3396.05 .35 .30$
}

\section{Abstract}

A natural language generation system is typically constituted by two main components: a content planning component (e.g., text planner or dialogue act planner) and a linguistic realization component. But, this is not sufficient since, on the one hand, the message built by the content planning component is generally not adequately detailed in order to control the many possibilities for its expression and, on the other hand, the content planner cannot influence the way in which the message will be verbalized.

Generation systems require a third component, called the micro-planning (or sentence planning or phrasing) component, which acts as an intermediary between the pragmatico-semantic level and the purely syntactic level. The micro-planner is responsible for transforming the message into a textual structure. For this transformation to be achieved, grammatical and lexical resources must be selected.

\section{Introduction}

Traditionally, the architecture of a generation system is either pipelined (e.g., TEXT [McKeown 85]), interleaved (e.g., PAULINE [Hovy 90]) or integrated (e.g., KAMP [Appelt 85]). Whatever the approach, the system is divided into two main components: a message planning component and a linguistic realization component. The former is responsible for the selection and organization of the information to be conveyed; it builds a structure often called message. The latter involves the formulation of the message in grammatically correct sentences. In a context of discourse, the message planning component is a text planner and the message is a text plan

\footnotetext{
*This work was realized at the Istituto per la Ricerca Scientifico e Tecnologica (IRST) in Trento (Italy).
}

(e.g., see [Maier \& Not 93]). In a context of dialogue, the message planner is a 'fictitious' component consisting of a dialogue act planner, which precedes the generation system, and a planner for surface linguistic acts, which is a component of the generation system. The dialogue act planner builds plans of dialogue acts. A plan of dialogue acts is a tree-like structure in which non-leaf nodes are macro-acts (e.g., "disjunction" or "sequence" of dialogue acts) and leaf nodes are dialogue acts (see [Sadek 91]). A dialogue act conveys information about its type (e.g., "inform", "yn-question" or "confirm"), its propositional content (i.e., a semantico-conceptual representation of the information to be communicated), and the interlocutors. The surface linguistic act planner transforms a plan of dialogue acts into a plan of surface linguistic acts (i.e., the message). In particular, the dialogue act type is transformed into a surface linguistic act type such as "assertion", "answer" or "question", and the semanticoconceptual content into a semantic propositional content (cf. figure 1 and section 3 ).

One of the problems which appear in traditional generation systems concerns the message itself: it is underspecified with respect to the language. On the one hand, the message planner cannot influence the way in which the message will be verbalized, and, on the other hand, the message is not detailed enough for the many possibilities for its expression to be controlled. Figure 1 shows several messages (a text plan and three surface linguistic acts corresponding to the same dialogue act) and their different verbalizations.

A second problem is related to the fact that most generation systems produce texts successfully since they implicitly ensure that the messages are expressible. For instance, in the case where the message is a text plan, each atomic unit in the message must be a proposition, and thus can always be realized as a clause. Each unit can be independently translated into the language using the linguistic realization component, since there are 
Text plan: (r1 / result

$$
\begin{gathered}
\text { nucleus (e1 / shoot } \\
\text { agent john } \\
\text { patient mary) } \\
\text { satellite (e2 / kill } \\
\text { agent john } \\
\text { patient mary)) }
\end{gathered}
$$

(A1) John shot Mary. He killed her.

$\left(\mathrm{A}_{2}\right)$ Mary was shot by John. He killed her.

$\left(\mathrm{A}_{3}\right)$ Mary was shot by John. She was killed.

$\left(\mathrm{A}_{4}\right)$ John shot Mary killing her.

(As) John shot Mary who was killed.

Dialogue act: $\langle S, \operatorname{Inform}(U, \iota x$ arrival-airport $(x, \iota y$ flight $(y) \wedge \operatorname{departure-time}(y, 10: 00))=J F K)>$

This dialogue act can be transformed into three distinct "assertions" or "answers".

- Content $1=$ a destination-event whose theme is "the 10:00 flight" and destination is "JFK":

$\left(\mathrm{B}_{A 1}\right)$ The 10:00 fight goes to JFK.

(B $\left.{ }_{A 2}\right)$ It goes to $J F K$.

(B $\left.{ }_{A 3}\right)$ To JFK.

- Content $2=$ an identity between "the arrival airport of the 10:00 flight" and "JFK":

$\left(\mathrm{B}_{B 1}\right)$ The arrival airport of the 10:00 flight is JFK.

$\left(\mathrm{B}_{B 2}\right)$ The arrival airport is $J F K$.

$\left(\mathrm{B}_{B 3}\right) J F K$.

- Content $3=$ a destination-event whose theme is "the hearer" and destination is "JFK":

$\left(\mathrm{B}_{C 1}\right)$ You will arrive at JFK.

$\left(\mathrm{B}_{C 2}\right)$ At JFK.

Figure 1: Messages and their possible verbalizations

few restrictions on how to connect clauses. In particular, clauses can be connected with coordinate or subordinate conjunctions or can be simply realized as separate sentences. However, this kind of approach does not use the full expressive power of the language in which units may be much more tightly composed. Moreover, as argued in [Hovy 92], a two-component generation system is not satisfactory in producing 'good quality' texts since too many phenomena are ignored or processed in a simplistic way, for example, anaphora planning, choice of lexical items, aggregation of clauses and noun phrases, selection of grammatical constructions (e.g., the choice of the verb voice), and segmentation into sentences (i.e., single sentence vs. separate sentences, hypotaxis vs. parataxis, and determination of clause order). The main difficulty in taking these phenomena into account is due to the fact that they are "supra-grammatical but not really related to content selection and organization" [Hovy 92, p. 2].

In fact, Hovy argues that these phenomena should be treated after the message planning and before the linguistic realization.

A third problem is related to the fact that in a twocomponent generation system the conceptual decisions are taken by the message planner and the the linguistic decisions by the realization component. Since conceptual and linguistic decisions are dependent on each other (see [Danlos 87]), the two components should be able to communicate with each other. On the one hand, the definition of such a communication is a complex task, due to the gap between the two activities (see [Meteer 92]). On the other hand, all the grammatical details that can be provided by a linguistic realizer may not be useful to take these decisions. We think that, in a first step, it is use- 


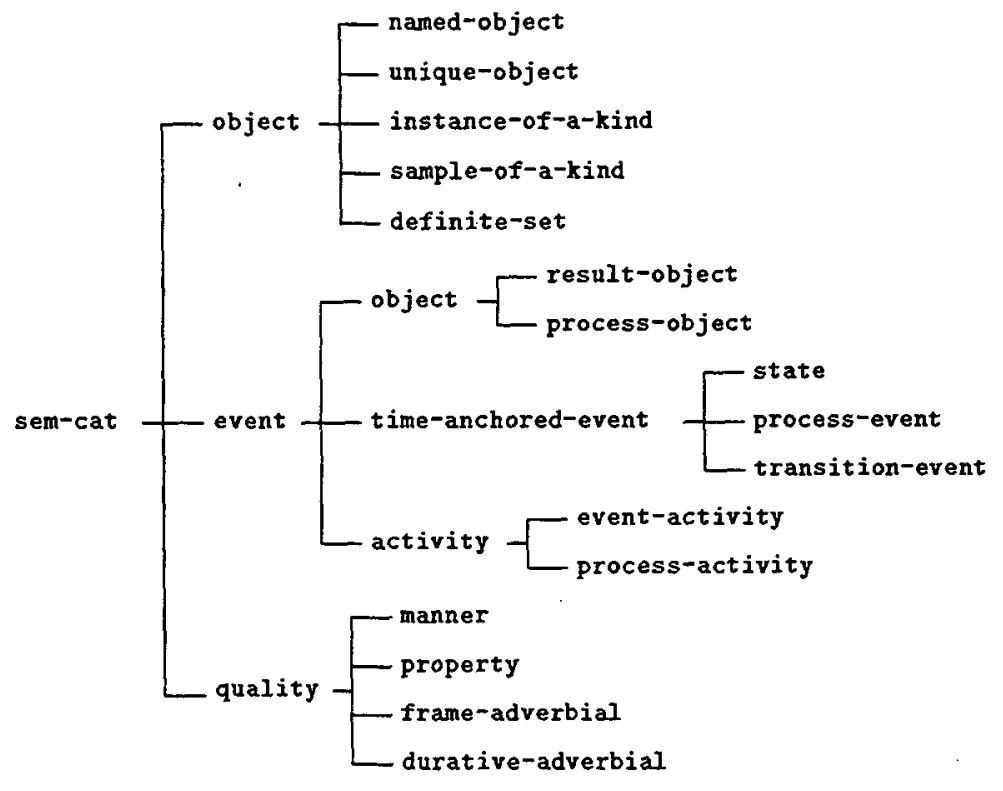

Figure 2: Partial hierarchy of semantic categories

ful to know that such linguistic phenomena exist in the target language without being able to realize them. For example, it is useful to know that a present participial clause can verbalize the rhetorical relation "result", but it is not useful to know (in a first step) that a present participial clause is formed by adding "ing" at the end of the stem of a verb.

\section{Micro-planning component}

In order to solve the above mentioned problems, a component can be added between the message planner and the linguistic realizer (e.g., see [Levelt 89] or [Hovy 92]). The role of this third component, called the micro-planner in [Levelt 89], is to plan the best informational perspective that can be given to the message. The component often uses an intermediate representation level. For example, in the system Epicure [Dale 88], a recoverable semantic structure, which specifies the underlying content of the utterance to be generated, and an abstract syntactic structure, which is much closer to the surface syntax of the linguistic form which will be produced, are used. Dale also shows how the two levels of representation play a role in the generation of elisions and one-anaphoric expressions. However, the abstract syntactic level of most generation systems is often hazy and dependent on the syntactic formalism used by the linguistic realization component.
Our intermediate structure, which we call textual ${ }^{1}$ structure, is expressed in terms of abstract linguistic resources [Meteer 92] ${ }^{2}$. These resources are abstractions over concrete resources of language (e.g., syntactic structures, words, and grammatical features); they reflect linguistic constraints while abstracting away from syntactic details. They are useful for generation systems since

allowing a generation system to select the concrete resources... makes available many more degrees of freedom than the language actually permits [Meteer 92, p. 67].

Abstract linguistic resources become the terms in which the micro-planning component takes its decisions and that way they constrain the system to only do what is expressible in the language since it is not allowed to choose them independently. We made some modifications on two kinds of abstract resources defined by Meteer: we improved her semantic categories by using perspectives from systemic functional linguistics (cf. section 2.1) and we extended the notion of resource trees (cf. section 2.2).

\footnotetext{
1"Textual" is used in the sense of the textual metafunction in the systemic functional theory (see below). For presentations of systemic functional linguistics, see [Halliday 85] or [Matthiessen \& Bateman 91].

${ }^{2}$ Meteer used the abstract linguistic resources to build a structure which she called text structure. We do not use the same name because Meteer's structure could exist at the same level of rhetorical structure theory (i.e., the message planning level) whereas our structure is complementary to this level.
} 
Moreover, we explicitly defined lexical resources (cf. section 2.3).

\subsection{Semantic hierarchies}

Semantic categories (cf. figure 2) reflect pertinent combinations of linguistic features (e.g., syntactic features and lexical items). For example, the five sub-categories of object ${ }^{3}$ are specified from definiteness (i.e., definite or indefinite) and number (i.e., singular or plural). These categories constrain the composition of the utterance by defining what kinds of constituents can be extended and how they can be composed. For example, an adjunct constituent of type manner can be added to an event (e.g., Peter suddenly decided to go to the beach) whereas an adjunct of type property cannot (e.g., "Peter importantly decided to go to the beach ${ }^{4}$ ); a process-event can be composed with a durative-adverbial (e.g., Karen watched TV for an hour) whereas a transition-event may be composed of a frame-adverbial (e.g., Karen solved the problem in one hour).

On the one hand, we think that according to systemic linguistic theory ${ }^{5}$, the hierarchy of semantic categories mixes ideational constraints and textual constraints; both are semantic, but semantic of rather different kinds. Thus, while it is correct that the categories event, manner, and property are semantic (ideational) categories, taking an event perspective on something is a textual decision. e.g.,

"The ship sank."

is an ideational event, and it is textually presented within an EVENT-PERSPECTIVE.

"The sinking of the ship"

is still an ideational event, but it is now presented within an OBJECT-PERSPECTIVE. Even clearer are cases such as definite and indefinite noun phrases. Thus the differences between "a man" and "the man" can be characterized as follows. They are both ideational of category PERSON. The noun phrase "a man" presents a discourse entity of that ideational semantic category which is textually UNIDENTIFIABLE. This is a category drawn from textual semantics. Textual semantic classifications of discourse entities change as a text develops. The noun phrase "the man" is classified textually as IDENTIFIABLE. The former is realized grammatically (largely on

\footnotetext{
${ }^{3}$ The semantic categories are noted in typerriter text.

${ }^{4}$ Following the general convention in linguistics, we use "*" to mark ungrammatical sentences.

E In this theory, language is organized functionally according to three metafunctions, concerned with the representation of experience (ideational), symbolic interaction between interlocutors (interpersonal), and presentation of information as text in context (textual).
}

the basis of the textual semantic classification) as an indefinite noun phrase, the latter as a definite noun phrase. In this way, ideational semantics and textual semantics are complementary to each other and both help constrain grammatical realization.

On the other hand, we think that the textual part of Meteer's hierarchy is too abstract to treat phenomena that need syntactic information such as anaphora planning and selection of grammatical constructions (see also the other examples of linguistic phenomena given in section 1); in particular, syntactic information such as gender or verb voice is missing.

In order to solve these problems, we propose to replace the hierarchy with two constituent hierarchies: an ideational semantic hierarchy and a textual semantic hierarchy. The former is based on the Upper Model defined in [Bateman et al. 90]. The latter was developed by ourselves; we call it the hierarchy of textual semantic categories. These two hierarchies are not incompatible since

[a] language... embodies a multiplicity of constituent hierarchies, coexisting in different parts of the system. They are not unrelated: on the contrary, they are all different facets of the same phenomenon. [...] At the same time, each of these hierarchies is independent of the others, and there are infinite possibilities of matching them up in a meaningful way [Halliday 85, p. 18 ].

The Upper Model provides a domain-independent, general and reusable conceptual organization which is used to classify the world knowledge when linguistic processing is to be performed. This general semantic taxonomy can also be seen as an inheritance hierarchy that organizes concepts according to how they can be expressed (cf. figure 3). The Upper Model also expresses (ideational) semantic restrictions on arguments required by entities it involves. For example, the realization-event ${ }^{6}$ requires two participants: an agent that must be an animate and a patient that must be a product. The Upper Model also provides the type of qualities which may be ascribed to particular concepts. For example, the Upper Model expresses that the destination relation relates a place object to an event.

The hierarchy of textual semantic categories is a property inheritance network that represents textual knowledge of the target language. Figure 4 shows a part of our hierarchy; the whole hierarchy contains about eighty categories. The definition of the textual semantic categories is based on an analysis of French [Bescherelle 90] and of English [Halliday 85, Shinghal 92].

\footnotetext{
${ }^{6}$ The ideational semantic categories are noted in italic text.
} 


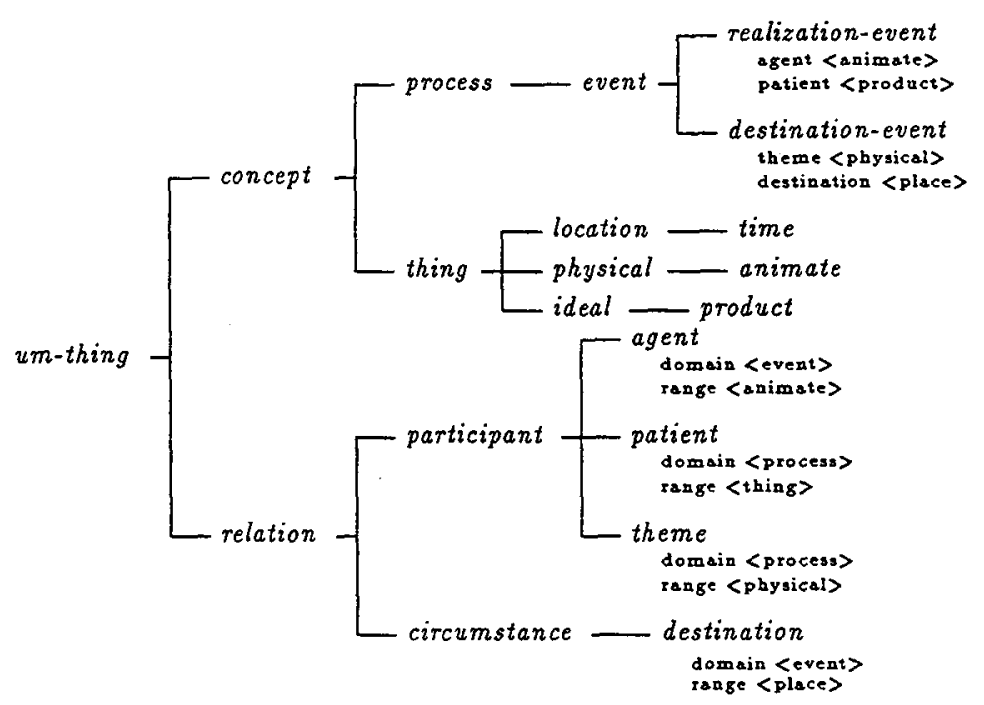

Figure 3: Partial Upper Model

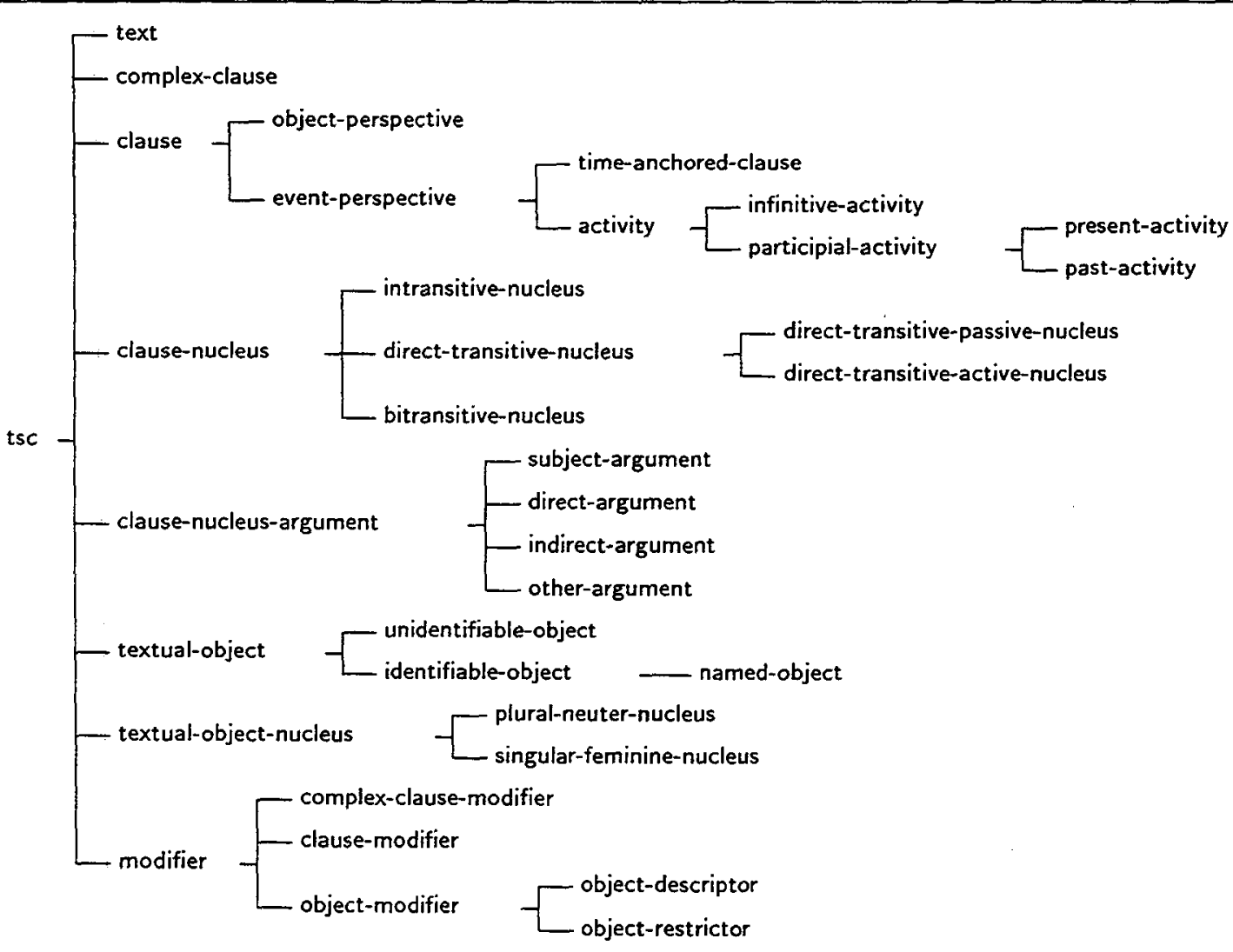

Figure 4: Partial textual semantic hierarchy 
We will explain the meaning of each textual category by describing the way in which they are realized from a grammatical viewpoint. Complex-clauses ${ }^{7}$ are realized as sentences. The sub-hierarchy of clause represents different linguistic forms of clause; in particular, the timeanchored-event is realized as a finite clause, the activity as a non finite clause and the object-perspective as clause nominalization. The clause-nucleus sub-hierarchy classifies different kinds of verbs (e.g., transitive and intransitive verbs) and verb voice. The other-argument is realized as verb argument that has no specific grammatical function, whereas the three other clause-nucleus-arguments are realized as constituents whose grammatical function is subject, direct object or indirect object. For example, the verb "to get to" requires two arguments: one subject-argument (realized as the subject) and one otherargument which represents the destination. The textualobject is realized as a noun phrase, and, in particular, the unidentifiable-object as an indefinite noun phrase and the identifiable-object as a definite one. The hierarchy of textual-object-nucleus corresponds to nouns; it represents various linguistic forms of nouns with respect to their gender and number. The modifiers are realized as adjectives, adverbs, prepositional phrases, and subordinate clauses.

\subsection{Resource trees}

Resource trees are primitive trees from which textual structures are built. In particular, they indicate the way in which a constituent (i.e., a textual structure node) can be expanded, that is, what are its sub-constituents, or extended, that is, what kind of additional sub-constituents can be added. They are defined from elementary trees. Meteer distinguished two basic forms of elementary trees: the kernel tree and the composite tree. These two trees represent Halliday's distinction between the multivariate and the univariate structure, respectively [Halliday 85]. The kernel tree (i.e., the HEAD-ARGUMENT structure) is an atomic structure, that means, it cannot be modified independently from the whole structure. Essentially, it represents a predicate/argument structure. The composite tree is an incremental structure, that is, new child nodes can be added at any time of the expansion of the mother node. Meteer distinguished the COMPOSITE-MATRIXADJUNCT structure, which corresponds to the hypotactic relation (i.e., the relation between dependent elements and their dominating node), and the COMPOSITECOORDINATE structure, which represents the paratactic relation (i.e., the relation between elements of equal status). Meteer defined resource trees as follows:

\section{(define-composite-tree complex-event-tree} (matrix \&rest adjunct)

\footnotetext{
${ }^{i}$ The textual semantic categories are noted in sans serif text.
}

:constructor-function build-complex-event-tree :matrix-restrictions . (state event) :adjunct-restrictions (manner location))

This definition expresses that the complex-event resource tree is a COMPOSITE tree whose MATRIX has to be of type state or event and ADJUNCTs of type manner or location. It also specifies a constructor function which builds the tree.

We think that the language for defining resource trees is not powerful enough. For instance, it must be explicitly specified that a composite tree only has one matrix node or that an activity does not have an explicit subject. In order to solve this problem, a more powerful language had to be defined. In particular, the restriction on daughter nodes is more precise. In addition, we introduce the notions of relevance and constraints. Restrictions are expressed by using a specific language which expresses quantities (e.g., "one" or "several") and description of the daughter constituents. In particular, each daughter constituent is described by a structure, which we call abstract constituent, which specifies the ideational and textual semantic categories of the constituent, its structural relationship with the mother node, and its relevance. The relevance is the link between textual structure nodes and parts of the message; it indicates the part of the message verbalized by the mother node and its child nodes. Constraints are conditions which must be satisfied by the textual structure. For example, constraints can express that the subject-argument of an infinitiveactivity will not explicitly appear in the final text. Figure 5 shows the definition of the "infinitive-activity" resource tree $^{8}$, which is a composite tree whose mother node has the textual category infinitive-activity. The tree has one and only one matrix node of the ideational category process and of the textual category clause-nucleus, and additional adjunct nodes of the ideational category circumstance and of the textual category clause-modifier. It specifies a constraint which states that the subject-argument must be implicit. It also indicates that the mother node and its daughter nodes correspond to the same part of the message, that is, they have the same relevance (cf. the variable $\mathrm{X}$ in figure 5$)^{9}$.

\subsection{Lexical resources}

Lexical items, i.e., words, are lexical resources of the language. We represent their meaning by associating each of them with entities of the Upper Model and of the tex-

\footnotetext{
${ }^{8}$ Meteer's resource trees are expressed in terms of semantic categories whereas our resource trees are defined in terms of textual categories.

9 The variable of the field "relevance" is unified with the relevance of a textual structure's node during the expansion of the node with the resource tree.
} 


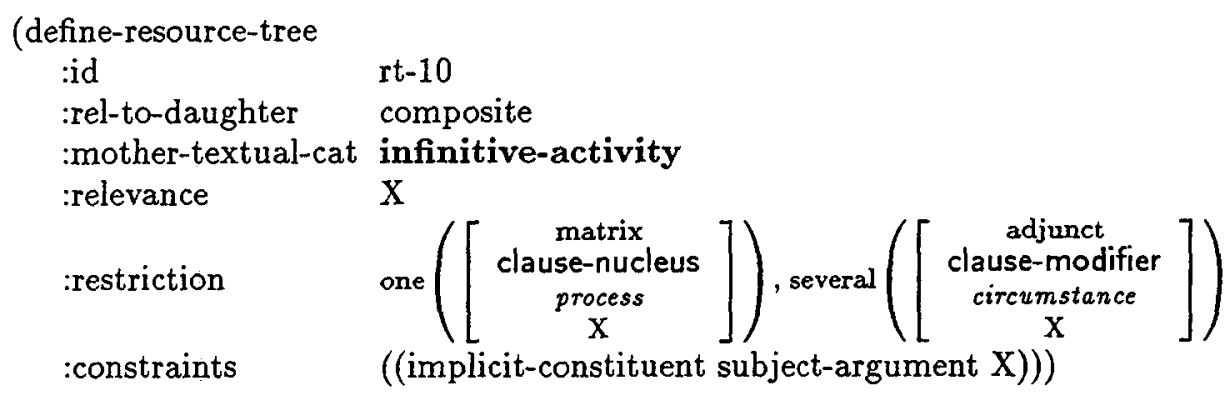

Figure 5: The "infinitive activity" resource tree

tual semantic hierarchy. For example, the lexical resource which corresponds to the direct transitive verb "to write" is defined as follows:

$$
\left[\begin{array}{l}
\text { "to-write" } \\
\text { (direct-transitive-nucleus) } \\
\text { (write-action) }
\end{array}\right]
$$

A lexical resource can be linked to more than one textual category; for example, the following resources

$$
\begin{aligned}
& {\left[\begin{array}{l}
\text { "article" } \\
\text { (singular-neuter-nucleus plural-neuter-nucleus) } \\
\text { (article) }
\end{array}\right]} \\
& {\left[\begin{array}{l}
\text { "quick" } \\
\text { (object-modifier clause-modifier) } \\
\text { (quick) }
\end{array}\right]}
\end{aligned}
$$

correspond to the nouns "article" and "articles" and to the adjective "quick" and the adverb "quickly", respectively. A lexical resource can also be associated with several ideational categories; for example, the following resource

$$
\left[\begin{array}{l}
\text { "so-that" } \\
\text { (clause-modifier) } \\
\text { (purpose enablement) }
\end{array}\right]
$$

can be used to verbalize a rhetorical relation "purpose" or "enablement".

\subsection{Textual structure}

The textual structure is a tree-like structure built from resource trees. It represents the constituency of the utterance (each node is a complete constituent of the utterance), the structural relations among constituents (head-argument, composite-matrix-adjunct, compositecoordinate), the ideational and textual categories, the relevance and the restriction of each node, and the lexemes.
In addition, a marker which describes the lexical cohesion of the text (see [Halliday \& Hasan 76]) is associated to each lexeme. The textual structure provides linguistic information which is required for treating specific linguistic phenomena such as anaphora planning (see section 1). For example, in figure 6 (only the main information contained in each node is depicted), the textual structure indicates that the domain instance "Stock" corresponds to a male and will be verbalized as a singular masculine proper name (named-object + singular-masculine-name).

Several textual structures can be built to verbalize one message. The structure which is selected is the one which has the higher cohesive score. This score is obtained by summing the scores of lexical cohesion of all lexical resources used in the textual structure. The score of each resource is obtained as follows:

- if the lexical resource appears in the local context (cf. history of dialogue acts) then the lexical cohesion is scored 3;

- if the lexical resource appears in the active set of the history of linguistic resources then the score is 2 ;

- if the lexical resource appears in the open set of the history of linguistic resources then the score is 1 ;

- otherwise, the score is 0 .

\section{Utterance generation in the context of dialogue}

A plan of dialogue acts is verbalized by transforming it into two successive representational levels: surface linguistic acts and textual representations. These two main transformations are achieved with respect to the current dialogue context. We represent the context through four histories. The history of dialogue acts stores, in particular, the requests of the interlocutor that have not been 


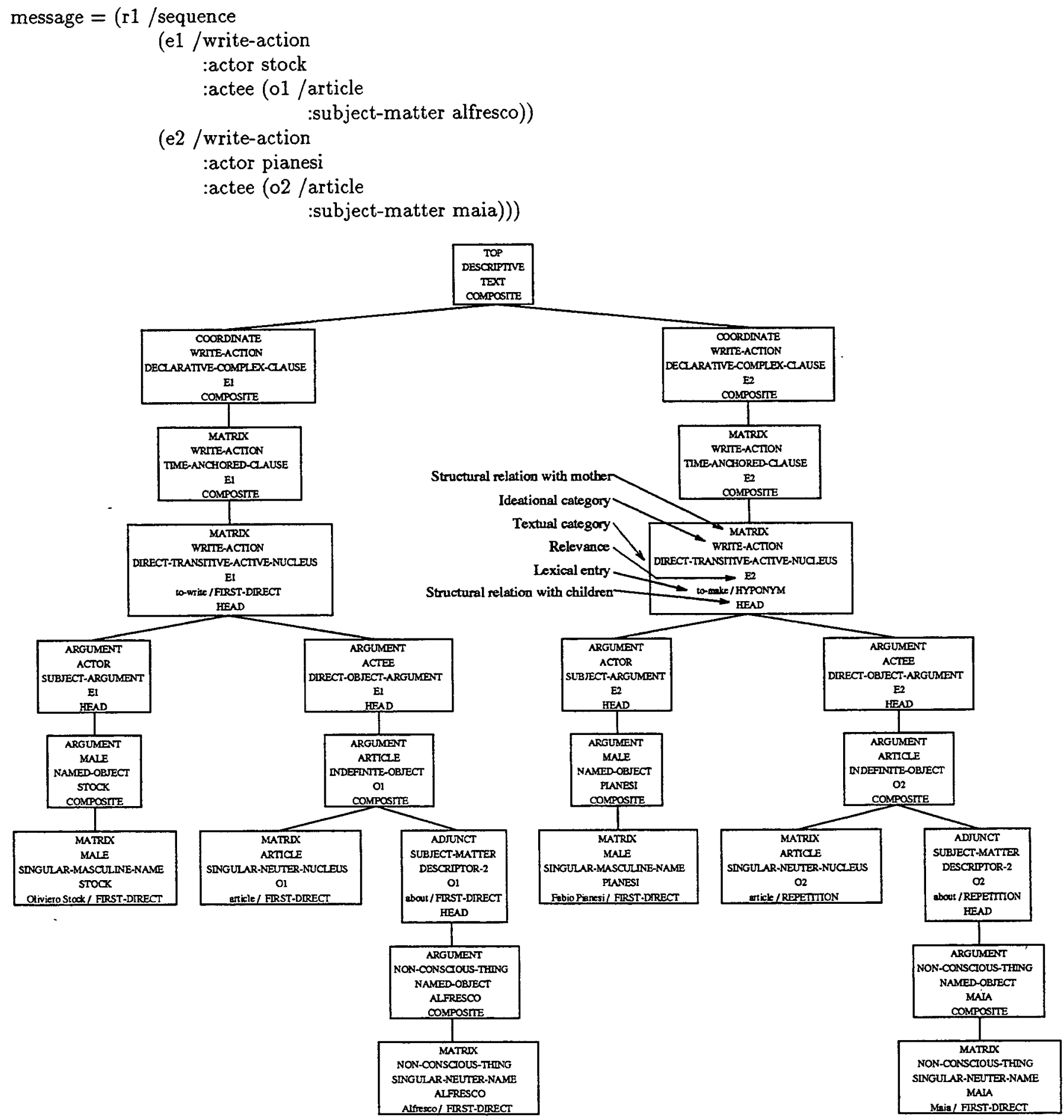

$\rightarrow$ "Oliviero Stock wrote an article about Alfresco. Fabio Pianesi made one about MAIA."

Figure 6: An example of textual structure 
answered yet. Each item of this list provides information on the dialogue act itself, the way in which conceptual entities that appear in its propositional content were realized with respect to the lexical semantics and the list of linguistic resources used. This information constitutes a local context during the verbalization of the answer. The history of conceptual entities contains information on the conceptual entities (e.g., "arrival-airport") already verbalized in the dialogue and the way in which they were realized with respect to lexical semantics. This history is used for the verbalization of all surface linguistic acts, except for "answers" (the semantic realization of conceptual entities that appear in an "answer" is constrained by the local context retrieved from the history of dialogue acts). The history of objects involves information on the instances of conceptual entities (e.g. "JFK") mentioned in the dialogue. It is used to retrieve objects that are required from the lexical semantics point of view during the surface linguistic act planning (cf. the ideational semantic restrictions on arguments of entities of the Upper Model). It also provides information that is useful during anaphora planning, such as the information status (given or new) of objects. Finally, the history of linguistic resources indicates which abstract linguistic resources have been used in the dialogue. It is divided into two sets: an active set and an open set. The active set contains the linguistic resources used in the last sentence of the dialogue, and the open set the resources used in the preceding sentences. In particular, this history is used to evaluate the cohesion of textual structures.

We will take an example to show how the generation system deals with these histories in order to verbalize a plan of dialogue acts. We will assume that the interlocutor (i.e., the user) uttered the following question during the dialogue:

\section{The 10:00 flight goes to which airport?}

The dialogue act planner builds a dialogue act of informing that the airport is "JFK" 10 (this act is shown in figure 1). Since there is a request in the history of dialogue acts that corresponds to the dialogue act, the surface linguistic act planner decides to build an "answer" surface linguistic act. Then, it uses the local context provided by this history in order to transform the semanticoconceptual content into a semantic content. In particular, it builds a propositional content expressing a destinationevent whose theme is "the 10:00 flight" and whose destination is "JFK" (cf. content 1 in figure 1). Finally, the constructed linguistic act is passed to the micro-planning component. This component builds at least two textual structures representing the cases where the destinationevent is verbalized by "to go" or "to arrive". Since "to go"

\footnotetext{
${ }^{10}$ The way in which dialogue acts and plans of dialogue acts are built is explained in [Sadek 91].
}

appears in the local context, the selected textual structure is the one corresponding to the following utterance:

\section{The 10:00 flight goes to JFK.}

According to the content of the history of objects, the micro-planner can introduce anaphoric expressions. In that case, the built textual structure corresponds to:

\section{It goes to JFK.}

Finally, using the history of linguistic resources, the planner can produce ellipsis, in which case, the textual structure built corresponds to:

\section{To JFK.}

\section{Concluding remarks}

In this paper we described a component of a natural language generation, called the micro-planner, which is situated between the message planner and the linguistic realization component. Its role is to bridge the gap between the two traditional components in order to avoid some of the problems which arise in two-component generation systems. In order to achieve this, the micro-planner transforms the message built by the message planner into an intermediate structure, which we call textual structure. This structure represents an abstract grammatical organization of the final text. In particular, it provides semantic and syntactic information, which is useful to handle linguistic phenomena such as anaphora planning. This new representational level is based on the notion of abstract linguistic resources [Meteer 92]. We made two main modifications. Firstly, Meteer's semantic category hierarchy was substituted by two distinct semantic hierarchies: an ideational semantic hierarchy (i.e., the Upper Model [Bateman et al. 90]) and a textual semantic hierarchy. Secondly, we increased the expressibility of the resource tree definition language. Moreover, we defined a simple mechanism to represent lexical resources of the language.

In a three-component generation system, the microplanner makes the linguistic decisions (e.g., the selection of syntactic structures and the choice of lexical items) that are usually taken by the linguistic realizer in a two-component generation system. The advantages are, firstly, taking a decision is simpler since the micro-planner deals with abstract linguistic knowledge, secondly, the micro-planner is closer to the message planner than the linguistic realizer, and, thirdly, the linguistic realization component is simplified since it no longer has to take linguistic decisions. Such a component appears in the generation system of [Danlos 87]: the strategic component takes conceptual and linguistic decisions, and the tactical component only applies grammar rules. In fact, the 
linguistic realization component only deals with syntactic details such as agreement and complete surface order and morphology.

Our micro-planning component is currently being integrated in a text generation system developed at IRST (see [Maier \& Not 93, Panaget 94]). A previous version ([Panaget 92]) was implemented in order to be integrated in a human-computer oral dialogue system developed at CNET [Sadek et al. 94] . For the former case, the linguistic realization component [Pianesi 91] is based on the government and binding theory and a head-driven bottomup generation algorithm. The component consists of an integrated system for editing, modifying and maintaining unification-based grammars. For the latter case, the linguistic realizer is based on Tree Adjoining Grammar [Joshi 87]. In particular, the textual structure is transformed into a derivation tree (i.e., a tree which specifies a derived tree through a bottom-up traversal), which is then 'executed' by the linguistic component in order to build a derived tree (i.e., a syntactic tree).

\section{Acknowledgements}

I am extremely grateful to John Bateman, Elisabeth Maier, Elena Not, David Sadek and Oliviero Stock for their helpful comments on early versions of this paper.

\section{References}

[Appelt 85] Appelt D.E., Planning english sentences; Cambridge University Press, 1985.

[Bateman et al. 90] Bateman J.A., Kasper R.T., Moore J.D. \& Whitney R.A., A general organization of the knowledge for natural language processing: the Penman Upper Model, ISI research report, California, 1990.

[Bescherelle 90] Bescherelle 3 - La grammaire pour tous, Hatier, 1990.

[Dale 88] Dale R., Generating referring expressions in a domain of objects and processes, PhD, University of Edinburgh, 1988.

[Danlos 87] Danlos L., The linguistics basis of text generation, Cambridge University Press, 1987.

[Halliday 85] Halliday M.A.K., Introduction to functional grammar, Edward Arnold, 1985.

[Halliday \& Hasan 76] Halliday M.A.K. \& Hasan R., Cohesion in english, Longman inc., New York, 1976.

[Hovy 90] Hovy E., Pragmatics and natural language generation, Artificial Intelligence 43(2), pp 153-197, 1990 .
[Hovy 92] Hovy E., Sentence planning requirements for automated explanation generation, DIAMOND Bericht $\mathrm{n}^{\circ} 23,1992$.

[Joshi 87] Joshi A.K., The relevance of Tree Adjoining Grammar to generation, In Kempen G. ed., Natural language generation, Dordrecht, Martinus Nijhoff, 1987.

[Levelt 89] Levelt W.J.M., Speaking - from intention to articulation, The MIT Press, 1989.

[Mckeown 85] Mc Keown K.R., Discourse strategies for generating natural language text, Artificial Intelligence 27(1), pp 1-41, 1985.

[Maier \& Not 93] Maier E. \& Not E., Increasing cohesion in automatically generated natural language texts, In Torasso P. ed., Advances in AI - Third congress of the Italian Association for AI, Lectures notes in AI 728, Springer-Verlag, 1993.

[Matthiessen \& Bateman 91] Matthiessen C. \& Bateman J.A., Text generation and systemic-functional linguistics - Experiences from English and Japanese, Pinter Publishers, London, 1991.

[Meteer 92] Meteer M., Expressibility and the problem of efficient text planning, Pinter publishers, 1992.

[Panaget 92] Panaget F., De l'acte de dialogue au langage natural: un générateur d'énoncés en contexte de dialogue oral, CNET Technical Report NT/LAA/TSS/440, Lannion, France, 1992.

[Panaget 94] Panaget F., The micro-planning component of a generation system, IRST Technical Report 940205, Trento, Italy, 1994.

[Pianesi 91] Pianesi F., Head driven bottom up generation and government and binding: a unified perspective, In proceedings of the $3^{r d}$ European Workshop on Natural Language Generation, Austria, 1991.

[Sadek 91] Sadek M.D., Dialogue acts are rational plans, In proceedings of the ESCA/ETRW workshop on "the structure of multimodal dialogue", Italy, September 16$20,1991$.

[Sadek et al. 94] Sadek M.D., Ferrieux A \& Cozannet A., Towards an artificial agent as the kernel of a spoken dialogue system: a progress report, To appear in the proceedings of the AAAI workshop on integration of natural language and speech processing, 1994.

[Shinghal 92] Shinghal R., Natural language processing: a prescriptive grammar, In Formal conceptions in Artificial Intelligence, pp 131-232, Chapman \& Hall, 1992. 